Bull. Korean Math. Soc. 50 (2013), No. 3, pp. 993-1005

http://dx.doi.org/10.4134/BKMS.2013.50.3.993

\title{
NOTE FOR THE TRIPLED AND QUADRUPLE FIXED POINTS OF THE MIXED MONOTONE MAPPINGS
}

\author{
Jun Wu AND Yicheng LiU
}

\begin{abstract}
In this paper, to include more generalized cases, the authors present a modified concept for the tripled and quadruple fixed point of the mixed monotone mappings. Also, they investigate the existence and uniqueness of fixed point of the ordered monotone operator with the Matkowski contractive conditions in the partial ordered metric spaces. As the direct consequences, the existence of coupled fixed point, tripled fixed point and quadruple fixed point are explored at the common framework and some previous results in $[T$. G. Bhaskar and V. Lakshmikantham, Fixed point theory in partially ordered metric spaces and applications, Nonlinear Anal. 65 (2006), 1379-1393; V. Berinde and M. Borcut, Tripled fixed point theorems for contractive type mappings in partially ordered metric spaces, Nonlinear Anal. 74 (2011), no. 15, 4889-4897; E. Karapinar and N. V. Luong, Quadruple fixed point theorems for nonlinear contractions, Computers and Mathematics with Applications (2012), doi:10.1016/j.camwa.2012.02061] are improved. Finally, some fixed point theorems are proved.
\end{abstract}

\section{Introduction}

The contraction fixed point theorem is a key and handy tool to explore the unknown situations in the real world. There are various extensive investigation for the fixed point theorems with contractive conditions in metric space, cone metric space, partially ordered metric space and so on. For the successful applications to the differential and integral equations, fixed point theorems for the mixed monotone mapping are speedy developed in recent decade. In 2006, Gnana-Bhaskar and Lakshmikantham [4] introduced the concept of coupled fixed point and proved some coupled fixed point theorems for the mixed monotone mappings. Recently, the notions of tripled fixed point and quadruple fixed point were introduced by the authors in literatures [3] and [12], respectively. Meanwhile, the corresponding fixed point theorems were proved. Also, the notion of $g$-monotone property was introduced by Lakshmikantham and

Received May 26, 2012; Revised July 31, 2012

2010 Mathematics Subject Classification. 47H10, 54H25.

Key words and phrases. contractive mapping, quadruple fixed point, mixed monotone mapping, fixed point. 
Ciric in [14] and the coincidence point theorem was shown. More details for the direction of the coupled fixed point theory and applications can be referred in $[1,2,5,7,8,9,10,11,13,15,18,19,20]$, and the references therein.

In this paper, to include more common cases, we present a modified concept for the tripled and quadruple fixed point for the mixed monotone mappings. Also, we investigate the existence and uniqueness of fixed point of the ordered monotone operator with the Matkowski contractive conditions in the partial ordered metric spaces. As the direct consequences, the existence of coupled fixed point, tripled fixed point and quadruple fixed point are explored at the common framework and some previous results are improved. Finally, some fixed point theorems are proved.

Let $(X, \leq)$ be a partially ordered set, the subset $E \subset X$ is said to be a totally ordered subset if either $x \leq y$ or $y \leq x$ holds for all $x, y \in E$. We say the elements $x$ and $y$ are comparable if either $x \leq y$ or $y \leq x$ holds. It is said that the triple $(X, \leq, d)$ is a partially (totally) ordered complete metric space if $(X, \leq)$ is a partially (totally) ordered set and $(X, d)$ is a complete metric space. It is said that the operator $F: X \rightarrow X$ is non-decreasing monotone with respect to the order $\leq$ if for any $x, y \in X$ with $x \leq y$ then $F(x) \leq F(y)$. Let $\Phi$ denote all the increasing functions $\phi:[0,+\infty) \rightarrow[0,+\infty)$ which satisfy that $\lim _{n \rightarrow+\infty} \phi^{n}(r)=0$ for all $r>0$.

Definition 1.1 (Bhaskar and Lakshmikantham [4]). Let $(X, \leq)$ be a partially ordered set and $F: X \times X \rightarrow X$. The mapping $F$ is said to has the mixed monotone property if $F$ is monotone non-decreasing in its first argument and is monotone nonincreasing in its second argument, that is, for any $x, y \in X$,

$$
\begin{aligned}
x_{1}, x_{2} \in X, x_{1} \leq x_{2} \Rightarrow F\left(x_{1}, y\right) \leq F\left(x_{2}, y\right) \text { and } \\
y_{1}, y_{2} \in X, y_{1} \leq y_{2} \Rightarrow F\left(x, y_{2}\right) \leq F\left(x, y_{1}\right) .
\end{aligned}
$$

Definition 1.2 (Bhaskar and Lakshmikantham [4]). An element $(x, y) \in X^{2}$ is said to be a coupled fixed point of the mapping $F: X^{2} \rightarrow X$ if $F(x, y)=x$ and $F(y, x)=y$.

Lemma $1.1([16,17])$. Let $\phi:[0,+\infty) \rightarrow[0,+\infty)$ be increasing and if for $t>0, \lim _{n \rightarrow+\infty} \phi^{n}(t)=0$. Then $\phi(t)<t$.

\section{Definitions of tripled and quadruple fixed point}

Berinde and Borcut [3] introduced the notion of mixed monotone property for the mappings from $X^{3}$ to $X$.

Definition $2.1([3])$. Let $(X, \leq)$ be a partially ordered set and $F: X^{3} \rightarrow X$ be a mapping. We say that $F$ has the mixed monotone property if $F(x, y, z)$ is monotone non-decreasing in $x$ and $z$, and it is monotone non-increasing in $y$, that is, for any $x, y, z \in X$ the implications below hold

$$
x_{1}, x_{2} \in X, x_{1} \leq x_{2} \Rightarrow F\left(x_{1}, y, z\right) \leq F\left(x_{2}, y, z\right)
$$




$$
\begin{aligned}
& y_{1}, y_{2} \in X, y_{1} \leq y_{2} \quad \Rightarrow \quad F\left(x, y_{2}, z\right) \leq F\left(x, y_{1}, z\right) \\
& z_{1}, z_{2} \in X, z_{1} \leq z_{2} \quad \Rightarrow \quad F\left(x, y, z_{1}\right) \leq F\left(x, y, z_{2}\right) .
\end{aligned}
$$

Also, Karapinar and Luong [12] introduced the following mixed monotone property for the mappings from $X^{4}$ to $X$.

Definition $2.2([12])$. Let $(X, \leq)$ be a partially ordered set and $F: X^{4} \rightarrow X$ be a mapping. We say that $F$ has the mixed monotone property if $F(x, y, z, w)$ is monotone non-decreasing in $x$ and $z$, and it is monotone non-increasing in $y$ and $w$, that is, for any $x, y, z, w \in X$ the implications below hold:

$$
\begin{aligned}
x_{1}, x_{2} \in X, x_{1} \leq x_{2} & \Rightarrow F\left(x_{1}, y, z, w\right) \leq F\left(x_{2}, y, z, w\right) \\
y_{1}, y_{2} \in X, y_{1} \leq y_{2} & \Rightarrow F\left(x, y_{2}, z, w\right) \leq F\left(x, y_{1}, z, w\right) ; \\
z_{1}, z_{2} \in X, z_{1} \leq z_{2} & \Rightarrow F\left(x, y, z_{1}, w\right) \leq F\left(x, y, z_{2}, w\right) \\
w_{1}, w_{2} \in X, w_{1} \leq w_{2} & \Rightarrow F\left(x, y, z, w_{2}\right) \leq F\left(x, y, z, w_{1}\right) .
\end{aligned}
$$

Following the concept in [3], an element $(x, y, z) \in X^{3}$ is called a tripled fixed point of $F: X^{3} \rightarrow X$ if $F(x, y, z)=x, F(y, x, y)=y$ and $F(z, y, x)=z$. Similarly, Karapinar and Luong [12] introduced the concept of quadruple fixed point: an element $(x, y, z, w) \in X^{4}$ is called a quadruple fixed point of $F: X^{4} \rightarrow$ $X$ if $F(x, y, z, w)=x, F(y, z, w, x)=y, F(z, w, x, y)=z$ and $F(w, x, y, z)=w$.

For a mapping $F: X^{3} \rightarrow X$, if there is an element $\left(x_{0}, y_{0}, z_{0}\right) \in X^{3}$ satisfying $F\left(x_{0}, y_{0}, z_{0}\right)=x_{0}, F\left(y_{0}, z_{0}, y_{0}\right)=y_{0}$ and $F\left(z_{0}, y_{0}, x_{0}\right)=z_{0}$, can we call the element $\left(x_{0}, y_{0}, z_{0}\right)$ a tripled fixed point of $F$ ? For the Berinde and Borcut's definition, it is invalid. Also, the concept quadruple fixed point suffered the same situation.

To avoid to suffer this situation, we introduce the modified concept of tripled and quadruple fixed point as follows.

Definition 2.3. An element $(x, y, z) \in X^{3}$ is called a tripled fixed point of $F: X^{3} \rightarrow X$ if

$$
F(x, y, z)=x,(F(y, x, y) \text { or }) F(y, z, y)=y \text { and } F(z, y, x)=z .
$$

Definition 2.4. An element $(x, y, z, w) \in X^{4}$ is called a quadruple fixed point of $F: X^{4} \rightarrow X$ if

$$
\begin{gathered}
(F(x, y, z, w) \text { or }) F(x, w, z, y)=x, \quad(F(y, z, w, x) \text { or }) F(y, x, w, z)=y \\
(F(z, w, x, y) \text { or }) F(z, y, x, w)=z \text { and }(F(w, x, y, z) \text { or }) F(w, z, y, x)=w .
\end{gathered}
$$

Definition 2.5. Let $k$ be an positive integer, an element $x \in X$ is called a fixed point of $F: X^{k} \rightarrow X$ if $F(x, x, \ldots, x)=x$.

\section{Auxiliary results}

The following two propositions can be found in the literature [6], but the proofs have some subtle differences. We put them for the sake of completeness. 
Proposition 3.1. Let $(X, \preceq, \rho)$ be a partially ordered complete metric space and $G: X \rightarrow X$ be a monotone non-decreasing operator with respect to the order $\preceq$ on $X$. Assume

(i) there is a $\varphi \in \Phi$ such that

$$
\rho(G(\tilde{x}), G(\tilde{y})) \leq \varphi(\rho(\tilde{x}, \tilde{y})) \text { for each } \tilde{x}, \tilde{y} \in X \text { with } \tilde{x} \preceq \tilde{y} .
$$

(ii) there exists an $\tilde{x}_{0} \in X$ such that $\tilde{x}_{0} \preceq G\left(\tilde{x}_{0}\right)$.

(iii) either (a) $G$ is a continuous operator or (b) if a nondecreasing monotone sequence $\tilde{x}_{n}$ in $X$ tends to $\bar{x}$, then $\tilde{x}_{n} \preceq \bar{x}$ for all $n$. Then the operator $G$ has a fixed point in $X$.

Proof. Definite a sequence $\left\{\tilde{x}_{n}\right\}$ in $X$ by

$$
\tilde{x}_{n}=G\left(\tilde{x}_{n-1}\right) \text { for } n=1,2, \ldots
$$

Considering $\tilde{x}_{0} \preceq G\left(\tilde{x}_{0}\right)$ and the operator $G$ is non-decreasing monotone with respect to the order $\preceq$, we have

$$
\tilde{x}_{0} \preceq \tilde{x}_{1} \preceq \tilde{x}_{2} \preceq \cdots \preceq \tilde{x}_{n} \preceq \cdots .
$$

If there exists $n_{0}$ such that $\tilde{x}_{n_{0}}=\tilde{x}_{n_{0}+1}$, then $\tilde{x}_{n_{0}}=G\left(\tilde{x}_{n_{0}}\right)$ and $\tilde{x}_{n_{0}}$ is a fixed point of $G$. Then the result of Proposition 3.1 trivially holds.

Suppose now that $\tilde{x}_{n} \neq \tilde{x}_{n+1}$ for all $n$. Setting $a_{n}=\rho\left(\tilde{x}_{n+1}, \tilde{x}_{n}\right)$, noting that the sequence $\left\{\tilde{x}_{n}\right\}$ is nondecreasing sequence in $X$, we have

$$
\begin{aligned}
a_{n+1} & =\rho\left(\tilde{x}_{n+2}, \tilde{x}_{n+1}\right)=\rho\left(G\left(\tilde{x}_{n+1}\right), G\left(\tilde{x}_{n}\right)\right) \\
& \leq \varphi\left(\rho\left(\tilde{x}_{n+1}, \tilde{x}_{n}\right)\right)=\varphi\left(a_{n}\right) \text { for } n=0,1, \ldots .
\end{aligned}
$$

Thus we obtain that

$$
0<a_{n+2} \leq \varphi\left(a_{n+1}\right) \leq \varphi^{2}\left(a_{n}\right) \leq \varphi^{3}\left(a_{n-1}\right) \leq \cdots \leq \varphi^{n+2}\left(a_{0}\right) .
$$

Since $\varphi \in \Phi$, we conclude that $\lim _{n \rightarrow \infty} \rho\left(\tilde{x}_{n+1}, \tilde{x}_{n}\right)=\lim _{n \rightarrow \infty} a_{n}=0$.

Now, we would prove that $\left\{\tilde{x}_{n}\right\}$ is a Cauchy sequence in $X$. In fact, for an arbitrary $\varepsilon>0$, since $\lim _{n \rightarrow \infty} \varphi^{n}(\varepsilon)=0$, it follows from Lemma 1.1 that $\varphi(\varepsilon)<\varepsilon$. So there is $N \in Z^{+}$such that $a_{N} \leq \varepsilon-\varphi(\varepsilon)$. Let $\tilde{x} \in \Omega:=\{\tilde{x} \in X$ : $\left.\rho\left(\tilde{x}, \tilde{x}_{N}\right) \leq \varepsilon, \tilde{x}_{N} \preceq \tilde{x}\right\}$, then by the triangle inequality

$$
\begin{aligned}
\rho\left(G(\tilde{x}), \tilde{x}_{N}\right) & \leq \rho\left(G(\tilde{x}), G\left(\tilde{x}_{N}\right)\right)+\rho\left(G\left(\tilde{x}_{N}\right), \tilde{x}_{N}\right) \\
& \leq \varphi\left(\rho\left(\tilde{x}, \tilde{x}_{N}\right)\right)+a_{N}<\varphi(\varepsilon)+a_{N} \\
& \leq \varphi(\varepsilon)+\varepsilon-\varphi(\varepsilon)=\varepsilon .
\end{aligned}
$$

Also, $\tilde{x}_{N} \preceq G\left(\tilde{x}_{N}\right) \preceq G(\tilde{x})$. This means that the set $\Omega$ is invariant for the operator $G$. Clearly, $\tilde{x}_{N} \in \Omega$. Thus $\tilde{x}_{N+p} \in \Omega$ for all $p \in Z^{+}$. So, the sequence $\left\{\tilde{x}_{n}\right\}$ is a Cauchy sequence in $X$. Since $(X, \rho)$ is a complete metric space, there exists a point $\bar{x} \in X$ such that $\lim _{n \rightarrow \infty} \tilde{x}_{n}=\bar{x}$.

Suppose that (a) in the assumption (iii) holds. Then by (2), we have

$$
\bar{x}=\lim _{n \rightarrow \infty} \tilde{x}_{n}=\lim _{n \rightarrow \infty} G\left(\tilde{x}_{n-1}\right)=G(\bar{x}) .
$$


Let us assume that the assumption (b) holds, then $\tilde{x}_{n} \preceq \bar{x}$ for all $n \in Z^{+}$. Thus from the assumption (i), we have

$$
\begin{aligned}
\rho(\bar{x}, G(\bar{x})) & \leq \rho\left(\bar{x}, G\left(\tilde{x}_{n}\right)\right)+\rho\left(G\left(\tilde{x}_{n}\right), G(\bar{x})\right) \\
& \leq \rho\left(\bar{x}, \tilde{x}_{n+1}\right)+\varphi\left(\rho\left(\tilde{x}_{n}, \bar{x}\right)\right) \rightarrow 0 \text { as } n \rightarrow \infty .
\end{aligned}
$$

So $\rho(\bar{x}, G(\bar{x}))=0$.

For both cases, the operator $G$ has a fixed point $\bar{x}$ in $X$. The proof of Proposition 3.1 is complete.

Let $D=\{\tilde{x} \in X: \tilde{x}$ and $G(\tilde{x})$ are comparable $\}$. Then we have:

Proposition 3.2. Let $(X, \preceq, \rho)$ be a partially ordered complete metric space and $G: X \rightarrow X$ be a monotone non-decreasing operator with respect to the order $\preceq$ on $X$. Assume (i) in Proposition 3.1 and one of following conditions hold:

(a) $G$ is a continuous operator;

(b) if a monotone sequence $\tilde{x}_{n}$ in $X$ tends to $\bar{x}$, then $\tilde{x}_{n}$ and $\bar{x}$ are comparable for all $n$.

Then the operator $G$ has a fixed point in $X$ if and only if $D \neq \phi$. Furthermore, if $D$ is a totally ordered nonempty subset, then the operator $G$ has a unique fixed point in $X$

Proof. It is easy to see that all the fixed points of $G$ fall in the set $D$. Thus if the operator $G$ has a fixed point in $X$, then $D \neq \phi$.

We suppose the condition (a) holds. If $D \neq \phi$ and $\tilde{x}_{0} \in D$, then there are two cases: $\tilde{x}_{0} \preceq G\left(\tilde{x}_{0}\right)$ or $G\left(\tilde{x}_{0}\right) \preceq \tilde{x}_{0}$. For the first case, following Proposition 3.1, we claim that the operator $G$ has a fixed point in $X$. For the other case: $G\left(\tilde{x}_{0}\right) \preceq \tilde{x}_{0}$, noting the symmetry of the metric, we see that the formula (1) holds for $\tilde{y} \preceq \tilde{x}$. Thus $\rho(G(\tilde{x}), G(\tilde{y})) \leq \varphi(\rho(\tilde{x}, \tilde{y}))$ for each $\tilde{x}, \tilde{y} \in X$ satisfying $\tilde{x}$ is comparable with $\tilde{y}$. Constructing the sequence $\left\{\tilde{x}_{n}\right\}$ in $X$ by $\tilde{x}_{n}=G\left(\tilde{x}_{n-1}\right)$ for $n=1,2, \ldots$, we have

$$
\cdots \preceq \tilde{x}_{n} \preceq \cdots \preceq \tilde{x}_{2} \preceq \tilde{x}_{1} \preceq \tilde{x}_{0} .
$$

Following the similar proof of Proposition 3.1 and resetting $\Omega:=\{\tilde{x} \in X$ : $\left.\rho\left(\tilde{x}, \tilde{x}_{N}\right) \leq \varepsilon, \tilde{x} \preceq \tilde{x}_{N}\right\}$, we conclude that the sequence $\left\{\tilde{x}_{n}\right\}$ tends to a fixed point of $G$.

Now we assume the condition (b) holds. Similar with the case (a), we see that the monotone sequence $\left\{\tilde{x}_{n}\right\}$ is a Cauchy sequence and label the limit point as $\bar{x}$. Thus $\bar{x}$ is comparable with $\tilde{x}_{n}$ for all $n \in Z^{+}$. Then we have

$$
\begin{aligned}
\rho(\bar{x}, G(\bar{x})) & \leq \rho\left(\bar{x}, G\left(\tilde{x}_{n}\right)\right)+\rho\left(G\left(\tilde{x}_{n}\right), G(\bar{x})\right) \\
& \leq \rho\left(\bar{x}, \tilde{x}_{n+1}\right)+\varphi\left(\rho\left(\tilde{x}_{n}, \bar{x}\right)\right) \rightarrow 0 \text { as } n \rightarrow \infty .
\end{aligned}
$$

Thus the operator $G$ has a fixed point $\bar{x}$ in $X$.

Next, we suppose that $D$ is a totally ordered nonempty subset. It is sufficient to prove the uniqueness of fixed point of $\tilde{F}$. Let $\tilde{x}$ and $\tilde{y}$ be two fixed points 
of $G$, then $\tilde{x}$ is comparable with $\tilde{y}, G(\tilde{x})=\tilde{x}$ and $G(\tilde{y})=\tilde{y}$. Following the assumption (i), we have

$$
\rho(\tilde{x}, \tilde{y})=\rho(G(\tilde{x}), G(\tilde{y})) \leq \varphi(\rho(\tilde{x}, \tilde{y})) \leq \varphi^{2}(\rho(\tilde{x}, \tilde{y})) \leq \cdots \leq \varphi^{n}(\rho(\tilde{x}, \tilde{y})) .
$$

Thus $\rho(\tilde{x}, \tilde{y})=0$, that is $\tilde{x}=\tilde{y}$. The proof of Proposition 3.2 is complete.

Let $\Phi_{1}$ denote all the functions $\phi:[0,+\infty) \rightarrow[0,+\infty)$ which satisfy that $\lim _{t \rightarrow r} \phi(t)>0$ for all $r>0$ and $\lim _{t \rightarrow 0+} \phi(t)=0$. Let $\Psi$ denote all the functions $\psi:[0,+\infty) \rightarrow[0,+\infty)$ which satisfy (i) $\psi(t)=0$ if and only if $t=0$, (ii) $\psi$ is continuous and non-decreasing, (iii) $\psi(s+t) \leq \psi(s)+\psi(t)$ for all $s, t \in[0,+\infty)$.

Lemma 3.1. If $\psi \in \Psi$ and $\phi \in \Phi_{1}$, then for $r \geq 0$,

$$
\varphi(r):=4 \sup _{0 \leq t \leq r}\left\{\psi^{-1}\left[\frac{1}{4} \psi(r)-\phi(r)\right]\right\}
$$

is well-defined and $\varphi \in \Phi$.

Proof. Since $\frac{1}{4} \psi(r) \leq \psi(r), \psi$ is continuous and (monotone) non-decreasing, $\psi^{-1}$ is well-defined. Obviously, the function $\varphi$ is increasing. Noting that $\psi(s+$ $t) \leq \psi(s)+\psi(t)$ for all $s, t \in[0,+\infty)$, we have

$$
\frac{1}{4} \psi(r)=\frac{1}{4} \psi\left(\frac{r}{4}+\frac{r}{4}+\frac{r}{4}+\frac{r}{4}\right) \leq \psi\left(\frac{r}{4}\right) \text { for all } r \geq 0 \text {. }
$$

Thus, for $r>0$, there is a $t_{0} \in(0, r]$, such that

$$
\begin{aligned}
\varphi(r) & =4 \lim _{t \rightarrow t_{0}} \psi^{-1}\left[\frac{1}{4} \psi(t)-\phi(t)\right] \\
& =4 \psi^{-1}\left[\frac{1}{4} \psi\left(t_{0}\right)-\lim _{t \rightarrow t_{0}} \phi(t)\right] \\
& <t_{0} \leq r .
\end{aligned}
$$

Then, for $r>0$, by

$$
0 \leq \varphi^{n}(r) \leq \varphi^{n-1}(r) \leq \cdots \leq \varphi(r) .
$$

Thus there is a constant $a \geq 0$ such that $\lim _{n \rightarrow \infty} \varphi^{n}(r)=a$. If $a>0$, by $\varphi^{n+2}(r)=\varphi\left(\varphi^{n+1}(r)\right) \leq \varphi^{n+1}(r)$, we see that $\lim _{r \rightarrow a+} \varphi(r)=a$. On the other hand, there is a $t_{a} \in(0, a]$ such that

$$
\begin{aligned}
\lim _{r \rightarrow a+} \varphi(r) & =4 \lim _{t \rightarrow t_{a}} \psi^{-1}\left[\frac{1}{4} \psi(t)-\phi(t)\right]=4 \psi^{-1}\left[\frac{1}{4} \psi\left(t_{a}\right)-\lim _{t \rightarrow t_{a}} \phi(t)\right] \\
& <4 \psi^{-1}\left[\frac{1}{4} \psi\left(t_{a}\right)\right] \leq t_{a} \leq a .
\end{aligned}
$$

This is a contradiction. Thus $\lim _{n \rightarrow \infty} \varphi^{n}(r)=0$ for all $r>0$, then $\varphi \in \Phi$.

Following Proposition 3.1, we have the next corollaries. 
Corollary 3.1 ([12], Theorem 11]). Let $(X, \leq)$ be a partially ordered set and $(X, d)$ be a complete metric space. Let $F: X^{4} \rightarrow X$ be a mapping having the mixed monotone property on $X$. Assume that for all $x \leq u, v \leq y, z \leq r$ and $t \leq w$

$$
\begin{aligned}
& \psi(d(F(x, y, z, w), F(u, v, r, t))) \\
\leq & \frac{1}{4} \psi(d(x, u)+d(y, v)+d(z, r)+d(w, t)) \\
& -\phi(d(x, u)+d(y, v)+d(z, r)+d(w, t)),
\end{aligned}
$$

where $\phi \in \Phi_{1}$ and $\psi \in \Psi$. Suppose that $F$ is a continuous mapping or $X$ has the following properties:

(i) if non-decreasing sequence $x_{n}$ tends to $x$, then $x_{n} \leq x$ for all $n$,

(ii) if non-increasing sequence $y_{n}$ tends to $y$, then $y \leq y_{n}$ for all $n$. If there exist $x_{0}, y_{0}, z_{0}, w_{0} \in X$ such that

$$
\begin{aligned}
& x_{0} \leq F\left(x_{0}, y_{0}, z_{0}, w_{0}\right), F\left(y_{0}, z_{0}, w_{0}, x_{0}\right) \leq y_{0}, \\
& z_{0} \leq F\left(z_{0}, w_{0}, x_{0}, y_{0}\right), F\left(w_{0}, x_{0}, y_{0} z_{0}\right) \leq w_{0},
\end{aligned}
$$

then there exist $x, y, z, w \in X$ such that

$F(x, y, z, w)=x$ and $F(y, z, w, x)=y, F(z, w, x, y)=z$ and $F(w, x, y, z)=w$.

Proof. Define the partial order $\preceq$ in $X^{4}$ be defined by

$$
P \preceq Q \Leftrightarrow x \leq u, v \leq y, z \leq r, t \leq w
$$

and $\rho(P, Q):=d(x, u)+d(y, v)+d(z, r)+d(w, t)$ for $P=(x, y, z, w), Q=$ $(u, v, r, t) \in X^{4}$. It is easy to see that $\left(X^{4}, \preceq, \rho\right)$ is a partially ordered complete metric space. Define the operator $G: X^{4} \rightarrow X^{4}$ by, for $P=(x, y, z, w)$,

$$
G(P)=(F(x, y, z, w), F(y, z, w, x), F(z, w, x, y), F(w, x, y, z)),
$$

then we would prove that $G$ is nondecreasing monotone operator on $X^{4}$ for the order $\preceq$. In fact, for $P_{1}=\left(x_{1}, y_{1}, z_{1}, w_{1}\right) \preceq P_{2}=\left(x_{2}, y_{2}, z_{2}, w_{2}\right)$, then by the mixed monotone property of $F$ on $X$ (see Definition 2.2), we have

$$
\begin{aligned}
F\left(x_{1}, y_{1}, z_{1}, w_{1}\right) & \leq F\left(x_{2}, y_{1}, z_{1}, w_{1}\right) \leq F\left(x_{2}, y_{1}, z_{2}, w_{1}\right) \\
& \leq F\left(x_{2}, y_{2}, z_{2}, w_{1}\right) \leq F\left(x_{2}, y_{2}, z_{2}, w_{2}\right) .
\end{aligned}
$$

Similarly, we have

$$
\begin{aligned}
& F\left(y_{2}, z_{2}, w_{2}, x_{2}\right) \leq F\left(y_{2}, z_{1}, w_{2}, x_{1}\right) \leq F\left(y_{1}, z_{1}, w_{1}, x_{1}\right), \\
& F\left(z_{1}, w_{1}, x_{1}, y_{1}\right) \leq F\left(z_{2}, w_{1}, x_{2}, y_{1}\right) \leq F\left(z_{2}, w_{2}, x_{2}, y_{2}\right), \\
& F\left(w_{2}, x_{2}, y_{2}, z_{2}\right) \leq F\left(w_{2}, x_{1}, y_{2}, z_{1}\right) \leq F\left(w_{1}, x_{1}, y_{1}, z_{1}\right) .
\end{aligned}
$$

Thus $G\left(P_{1}\right) \preceq G\left(P_{2}\right)$. This implies that $G$ is a nondecreasing monotone operator on $X^{4}$ for the order $\preceq$.

On the other hand, by Lemma 3.1, there is a $\varphi \in \Phi$ such that

$$
\begin{aligned}
\rho(G(P), G(Q))= & d(F(x, y, z, w), F(u, v, r, t))+d(F(z, w, x, y), F(r, t, u, v)) \\
& +d(F(y, x, w, z), F(v, u, t, r))+d(F(w, z, y, x), F(t, r, v, u))
\end{aligned}
$$




$$
\begin{aligned}
& \leq 4 \psi^{-1}\left[\frac{1}{4} \psi(\rho(P, Q))-\phi(\rho(P, Q))\right] \\
& =\varphi(\rho(P, Q)) .
\end{aligned}
$$

Also, there is a $P_{0}=\left(x_{0}, y_{0}, z_{0}, w_{0}\right) \in X^{4}$ such that $P_{0} \preceq G\left(P_{0}\right)$. Meanwhile, if the nondecreasing sequence $P_{n}=\left(x_{n}, y_{n}, z_{n}, w_{n}\right) \in X^{4}$ tends to the $P=$ $(x, y, z, w) \in X^{4}$, then $x_{n}$ and $z_{n}$ are non-decreasing in $X$ tend to $x$ and $z, y_{n}$ and $w_{n}$ are non-increasing in $X$ tend to $y$ and $w$, respectively. Thus by the property (i) and (ii), we have $P_{n} \preceq P$.

Following all above arguments, we see that the assumptions in Proposition 3.1 hold. Thus the operator $G$ has a fixed point $P=(x, y, z, w) \in X^{4}$, that is,

$$
(x, y, z, w)=(F(x, y, z, w), F(y, z, w, x), F(z, w, x, y), F(w, x, y, z)) .
$$

Thus we have

$$
F(x, y, z, w)=x, F(y, z, w, x)=y, F(z, w, x, y)=z \text { and } F(w, x, y, z)=w .
$$

The proof of Corollary 3.1 is complete.

Corollary 3.2. If the assumption (4) is replaced by there exist constants $c_{i} \in$ $[0,1)(i=1,2,3,4)$ such that $c_{1}+c_{2}+c_{3}+c_{4}<1$ and

$$
\left.d(F(x, y, z, w), F(u, v, r, t)) \leq c_{1} d(x, u)+c_{2} d(y, v)+c_{3} d(z, r)+c_{4} d(w, t)\right)
$$

for all $x \leq u, v \leq y, z \leq r$ and $t \leq w$, then the results in Corollary 3.1 are true.

Proof. Similar arguments as the proof of Corollary 3.1, let $\varphi(r)=\left(c_{1}+c_{2}+\right.$ $\left.c_{3}+c_{4}\right) r$ for $r \geq 0$ and re-estimate the formula

$$
\begin{aligned}
\rho(G(P), G(Q))= & d(F(x, y, z, w), F(u, v, r, t))+d(F(z, w, x, y), F(r, t, u, v)) \\
& +d(F(y, x, w, z), F(v, u, t, r))+d(F(w, z, y, x), F(t, r, v, u)) \\
\leq & \left(c_{1}+c_{2}+c_{3}+c_{4}\right) \rho(P, Q)=\varphi(\rho(P, Q))
\end{aligned}
$$

for $P, Q \in X^{4}$. Thus the results in Corollary 3.1 are true.

Corollary $3.3([3])$. Let $(X, \leq)$ be a partially ordered set and $(X, d)$ be a complete metric space. Let $F: X^{3} \rightarrow X$ be a mapping having the mixed monotone property on $X$. Assume that there exist constants $a, b, c \in[0,1)$ such that $a+b+c<1$ for which

$$
d(F(x, y, z), F(u, v, w)) \leq a d(x, u)+b d(y, v)+c d(z, w)
$$

for all $x \leq u, v \leq y, z \leq w$. Assume that $F$ is a continuous mapping or $X$ has the following properties:

(i) if non-decreasing sequence $x_{n}$ tends to $x$, then $x_{n} \leq x$ for all $n$,

(ii) if non-increasing sequence $y_{n}$ tends to $y$, then $y \leq y_{n}$ for all $n$. If there exist $x_{0}, y_{0}, z_{0} \in X$ such that

$$
x_{0} \leq F\left(x_{0}, y_{0}, z_{0}\right), F\left(y_{0}, x_{0}, y_{0}\right) \leq y_{0}, z_{0} \leq F\left(z_{0}, y_{0}, x_{0}\right),
$$

then there exist $x, y, z \in X$ such that

$$
F(x, y, z)=x \text { and } F(y, x, y)=y \text { and } F(z, y, x)=z .
$$


Proof. For a given constant $r \in(a+b+c, 1), R=(x, y, z) \in X^{3}$ and $S=$ $(u, v, w) \in X^{3}$, define

$$
\rho(R, S):= \begin{cases}(r-a) d(x, u)+(r-a) d(y, v)+(r-a-b) d(z, w) & \text { as } c=0, \\ (b+c) d(x, u)+(b+2 c) d(y, v)+c d(z, w) & \text { as } c \neq 0,\end{cases}
$$

then $\left(X^{3}, \preceq, \rho\right)$ is a partially ordered complete metric space, where the order $\preceq$ is given by

$$
R \preceq S \Leftrightarrow x \leq u, v \leq y, z \leq w .
$$

Also, define the operator $G: X^{3} \rightarrow X^{3}$ by

$$
G(R)=(F(x, y, z), F(y, x, y), F(z, y, x)) \text { for } R=(x, y, z) \in X^{3} .
$$

By the mixed monotone property of $F$, we conclude that $G$ is monotone nondecreasing for the order $\preceq$. Then there exists an $R_{0}=\left(x_{0}, y_{0}, z_{0}\right) \in X^{3}$ such that $R_{0} \preceq G\left(R_{0}\right)$.

On the other hand, as $c=0$, we have

$$
\begin{aligned}
& \rho(G(R), G(S)) \\
= & (r-a) d(F(x, y, z), F(u, v, w))+(r-b) d(F(y, x, y), F(v, u, v)) \\
& +(r-a-b) d(F(z, y, x), F(w, v, u)) \\
\leq & (r-a)[a d(x, u)+b d(y, v)+c d(z, w)] \\
& +(r-a)[a d(y, v)+b d(x, u)+c d(y, v)] \\
& +(r-a-b)[c d(x, u)+b d(y, v)+a d(z, w)] \\
\leq & r[(r-a) d(x, u)+(r-a) d(y, v)+(r-a-b) d(z, w)] \\
= & r \rho(R, S) .
\end{aligned}
$$

For $c \neq 0$, we have

$$
\begin{aligned}
& \rho(G(R), G(S)) \\
= & (b+c) d(F(x, y, z), F(u, v, w))+(b+2 c) d(F(y, x, y), F(v, u, v)) \\
& +c d(F(z, y, x), F(w, v, u)) \\
\leq & (b+c)[a d(x, u)+b d(y, v)+c d(z, w)] \\
& +(b+2 c)[a d(y, v)+b d(x, u)+c d(y, v)] \\
& +c[c d(x, u)+b d(y, v)+a d(z, w)] \\
\leq & r[b+c) d(x, u)+(b+2 c) d(y, v)+c d(z, w)] \\
= & r \rho(R, S) .
\end{aligned}
$$

Thus $\rho(G(R), G(S)) \leq r \rho(R, S)$. Taking $\varphi(t)=r t$ for $t>0$, from Proposition 3.1 , we conclude that $G$ has a fixed point $R=(x, y, z) \in X^{3}$. Thus there exist $x, y, z \in X$ such that

$$
F(x, y, z)=x, F(y, x, y)=y \text { and } F(z, y, x)=z .
$$


Remark 3.1. Additionally, in Corollary 3.3, if $F\left(y_{0}, z_{0}, y_{0}\right) \leq y_{0}$ holds. We redefine the operator $G: X^{3} \rightarrow X^{3}$ by

$$
G(\tilde{a})=(F(x, y, z), F(y, z, y), F(z, y, x)) \text { for } \tilde{a}=(x, y, z) \in X^{3},
$$

then it follows from the proof of Corollary 3.3 that there exist $x, y, z \in X$ such that

$$
F(x, y, z)=x, F(y, z, y)=y \text { and } F(z, y, x)=z
$$

\section{Fixed point theorems}

Theorem 4.1. Let $(X, \leq)$ be a totally ordered set and $(X, d)$ be a complete metric space. Let $F: X^{3} \rightarrow X$ be a mapping having the mixed monotone property on $X$. Assume that there exist constants $a, b, c \in[0,1)$ such that $a+b+c<1$ for which

$$
d(F(x, y, z), F(u, v, w)) \leq a d(x, u)+b d(y, v)+c d(z, w)
$$

for all $x \leq u, v \leq y, z \leq w$. Assume that $F$ is a continuous mapping or $X$ has the following properties:

(i) if non-decreasing sequence $x_{n}$ tends to $x$, then $x_{n} \leq x$ for all $n$,

(ii) if non-increasing sequence $y_{n}$ tends to $y$, then $y \leq y_{n}$ for all $n$. If there exist $x_{0}, y_{0}, z_{0} \in X$ such that one of following assumptions holds:

(a) $x_{0} \leq F\left(x_{0}, y_{0}, z_{0}\right), z_{0} \leq F\left(z_{0}, y_{0}, x_{0}\right)$ and $F\left(y_{0}, x_{0}, y_{0}\right) \leq y_{0}$;

(b) $x_{0} \leq F\left(x_{0}, y_{0}, z_{0}\right), z_{0} \leq F\left(z_{0}, y_{0}, x_{0}\right)$ and $F\left(y_{0}, z_{0}, y_{0}\right) \leq y_{0}$,

then $F$ has a unique fixed point in $X$, that is, there exists a unique $x \in X$ such that $F(x, x, x)=x$.

Proof. We suppose $F\left(y_{0}, z_{0}, y_{0}\right) \leq y_{0}$ hold. Basing the Corollary 3.3 and Remark 3.1, we conclude that there exist $x, y, z \in X$ such that

$$
F(x, y, z)=x, F(y, z, y)=y \text { and } F(z, y, x)=z .
$$

Next we prove $z=x$. In fact, by direct computation, we have

$$
\begin{aligned}
d(z, x) & =d(F(z, y, x), F(x, y, z)) \\
& \leq d(F(z, y, x), F(x, y, x))+d(F(x, y, x), F(x, y, z)) \\
& \leq a d(z, x)+c d(z, x) \\
& =(a+c) d(z, x) .
\end{aligned}
$$

Thus $d(z, x)=0$. Then we have $F(x, y, z)=F(x, y, x)=x$.

On the other hand,

$$
\begin{aligned}
d(y, x) & =d(F(y, x, y), F(x, y, x)) \\
& \leq d(F(y, x, y), F(x, x, x))+d(F(x, x, x), F(x, y, x)) \\
& \leq(a+c) d(y, x)+b d(y, x) \\
& =(a+b+c) d(y, x) .
\end{aligned}
$$


It follows from $a+b+c<1$ that $d(y, x)=0$. Then we have $x=y$. Thus there exists $x \in X$ such that $F(x, x, x)=x$.

Uniqueness: Let $x, y \in X$ satisfy $F(x, x, x)=x$ and $F(y, y, y)=y$. Since

$$
\begin{aligned}
d(x, y) & =d(F(x, x, x), F(y, y, y)) \\
& \leq d(F(x, x, x), F(y, x, y))+d(F(y, x, y), F(y, y, y)) \\
& \leq(a+b+c) d(x, y),
\end{aligned}
$$

we obtain $d(x, y)=0$. Then we conclude that there exists a unique $x \in X$ such that $F(x, x, x)=x$.

Theorem 4.2. Let $(X, \leq)$ be a totally ordered set and $(X, d)$ be a complete metric space. Let $F: X^{4} \rightarrow X$ be a mapping having the mixed monotone property on $X$. Assume that there exist constants $a, b, c, e \in[0,1)$ such that $a+b+c+e<1$ for which

$$
d(F(x, y, z, w), F(u, v, r, t)) \leq a d(x, u)+b d(y, v)+c d(z, r)+e d(w, t)
$$

for all $x \leq u, v \leq y, z \leq r, t \leq w$. Assume that $F$ is a continuous mapping or $X$ has the following properties:

(i) if non-decreasing sequence $x_{n}$ tends to $x$, then $x_{n} \leq x$ for all $n$,

(ii) if non-increasing sequence $y_{n}$ tends to $y$, then $y \leq y_{n}$ for all $n$. If there exist $x_{0}, y_{0}, z_{0}, w_{0} \in X$ such that

$$
\begin{gathered}
x_{0} \leq F\left(x_{0}, y_{0}, z_{0}, w_{0}\right), F\left(y_{0}, z_{0}, w_{0}, x_{0}\right) \leq y_{0}, \\
z_{0} \leq F\left(z_{0}, w_{0}, x_{0}, y_{0}\right), F\left(w_{0}, x_{0}, y_{0} z_{0}\right) \leq w_{0},
\end{gathered}
$$

then $F$ has a unique fixed point in $X$, that is, there exists a unique $x \in X$ such that $F(x, x, x, x)=x$.

Proof. It follows from Corollary 3.2 that there exist $x, y, z, w \in X$ such that

$$
F(x, y, z, w)=x, F(y, z, w, x)=y, F(z, w, x, y)=z, F(w, x, y z)=w .
$$

Since

$$
\begin{aligned}
d(z, x)= & d(F(z, w, x, y), F(x, y, z, w)) \\
\leq & d(F(z, w, x, y), F(x, w, x, y))+d(F(x, w, x, y), F(x, w, z, y)) \\
& +d(F(x, w, z, y), F(x, y, z, y))+d(F(x, y, z, y), F(x, y, z, w)) \\
\leq & a d(z, x)+c d(z, x)+b d(y, w)+e d(y, w),
\end{aligned}
$$

then we have $d(z, x) \leq \frac{b+e}{1-a-c} d(y, w)$.

Symmetrically, we can deduce that $d(y, w) \leq \frac{b+e}{1-a-c} d(z, x)$.

It follows from $a+b+c+e<1$ that $d(y, w)=d(z, x)=0$. Thus $z=x$ and $y=w$.

Furthermore, we have

$$
\begin{aligned}
d(x, y) & =d(F(x, y, x, y), F(y, x, y, x)) \\
& \leq d(F(x, y, x, y), F(x, x, x, x))+d(F(x, x, x, x), F(y, x, y, x))
\end{aligned}
$$




$$
\leq(a+b+c+e) d(x, y) .
$$

Thus $x=y$. The uniqueness is obvious. Then we can conclude that there exists a unique $x \in X$ such that $F(x, x, x, x)=x$. The proof of Theorem 4.2 is complete.

Exmple 4.1. Let $X=[0,+\infty), d(x, y)=|x-y|$ and define $F: X^{3} \rightarrow X$ by

$$
F(x, y, z)=\frac{1}{2} x+\frac{1}{5+y}+\frac{1}{4} z+2 .
$$

It is easy to see that $F$ is a mapping having the mixed monotone property and

$$
d(F(x, y, z), F(a, b, c)) \leq \frac{1}{2} d(a, x)+\frac{1}{5} d(b, y)+\frac{1}{4} d(c, z) .
$$

Also, there exist $x_{0}=z_{0}=1$ and $y_{0}=10$ such that $x_{0} \leq F\left(x_{0}, y_{0}, z_{0}\right), z_{0} \leq$ $F\left(z_{0}, y_{0}, x_{0}\right)$ and $F\left(y_{0}, x_{0}, y_{0}\right) \leq y_{0}$. Since $X$ is a totally ordered set, it follows from Theorem 4.1 that there is a unique $x=\frac{1}{2}(3+\sqrt{185})$ such that $F(x, x, x)=$ $x$.

Remark 4.1. If we replace the initial condition (5) in Theorem 4.2 by

$$
\begin{gathered}
x_{0} \leq F\left(x_{0}, w_{0}, z_{0}, y_{0}\right), F\left(y_{0}, x_{0}, w_{0}, z_{0}\right) \leq y_{0}, \\
z_{0} \leq F\left(z_{0}, w_{0}, x_{0}, y_{0}\right), F\left(w_{0}, x_{0}, y_{0} z_{0}\right) \leq w_{0},
\end{gathered}
$$

then $F$ also has a unique fixed point in $X$.

Acknowledgments. The authors are grateful to the reviewer for his/her valuable comments and suggestions. The second author supported by Hunan Provincial Natural Science Foundation of China (11JJ4007).

\section{References}

[1] A. Amini-Harandi and H. Emami, A fixed point theorem for contraction type maps in partially ordered metric spaces and application to ordinary differential equations, Nonlinear Anal. 72 (2010), no. 5, 2238-2242.

[2] H. Aydi, M. Postolache, and W. Shatanawi, Coupled fixed point results for $(\psi, \phi)$-weakly contractive mappings in ordered G-metric spaces, Comput. Math. Appl. 63 (2012), no. 1, 298-309.

[3] V. Berinde and M. Borcut, Tripled fixed point theorems for contractive type mappings in partially ordered metric spaces, Nonlinear Anal. 74 (2011), no. 15, 4889-4897.

[4] T. G. Bhaskar and V. Lakshmikantham, Fixed point theorems in partially ordered metric spaces and applications, Nonlinear Anal. 65 (2006), no. 7, 1379-1393.

[5] Y. Z. Chen, Existence theorems of coupled fixed points, J. Math. Anal. Appl. 154 (1991), no. $1,142-150$.

[6] J. Jachymski, Equivalent conditions for generalized contractions on (ordered) metric spaces, Nonlinear Anal. 74 (2011), no. 3, 768-774.

[7] E. Karapinar, Couple fixed point theorems for nonlinear contractions in cone metric spaces, Comput. Math. Appl. 59 (2010), no. 12, 3656-3668.

[8] - Quadruple fixed point theorems for weak $\phi$-contractions, ISRN Math. Anal. 2011 (2011), Article ID 989423, 16 pages.

[9] — A new quartet fixed point theorem for nonlinear contractions, J. Fixed Point Theory Appl. 6 (2011), no. 2, 119-135. 
[10] E. Karapinar and V. Berinde, Quadruple fixed point theorems for nonlinear contractions in partially ordered metric spaces, Banach J. Math. Anal. 6 (2012), no. 1, 74-89.

[11] _ Quadruple fixed point theorems for nonlinear contractions in partially ordered metric spaces, Banach J. Math. Anal. 6 (2012), no. 1, 74-89.

[12] E. Karapinar and N. V. Luong, Quadruple fixed point theorems for nonlinear contractions, Computers and Mathematics with Applications 2010 (2012), Comput. Math. Appl. 64 (2012), no. 6, 1839-1848.

[13] E. Karapinar, W. Shatanawi, and Z. Mustafa, Quadruple fixed point theorems under nonlinear contractive conditions in partially ordered metric spaces, Journal of Applied Mathematics 2012 (2012), Article ID 951912, 17 pages.

[14] V. Lakshmikantham and L. Ciric, Coupled fixed point theorems for nonlinear contractions in partially ordered metric spaces, Nonlinear Anal. 70 (2009), no. 12, 4341-4349.

[15] Y. Liu and Z. Li, Coincidence point theorems in probabilistic and fuzzy metric spaces, Fuzzy Sets and Systems 158 (2007), no. 1, 58-70.

[16] J. Matkowski, Integrable solutions of functional equations, Dissertationes Math. 127 (1975), 1-68.

[17] Amer. Math. Soc. 62 (1977), no. 2, 344-348.

[18] W. Shatanawi, Partially ordered cone metric spaces and coupled fixed point results, Comput. Math. Appl. 60 (2010), no. 8, 2508-2515.

[19] W. Shatanawi and B. Samet, On $(\psi, \phi)$-weakly contractive condition in partially ordered metric spaces, Comput. Math. Appl. 62 (2011), no. 8, 3204-3214.

[20] J. Z. Xiao, X. H. Zhu, and Y. F. Cao, Common coupled fixed point results for probabilistic $\phi$-contractions in Menger spaces, Nonlinear Anal. 74 (2011), no. 13, 4589-4600.

JuN Wu

College of Mathematics and Computer Science

Changsha University of Science Technology

Changsha, 410114, P. R. China

E-mail address: junwmath@hotmail.com

YICHENG LIU

Department of Mathematics and Systems Science

College of Science

National University of Defense Technology

Changsha, 410073, P. R. China

E-mail address: liuyc2001@hotmail.com 